\title{
Video Article \\ Analyzing Dynamic Protein Complexes Assembled On and Released From Biolayer Interferometry Biosensor Using Mass Spectrometry and Electron Microscopy
}

\author{
Alexandra J. Machen ${ }^{1}$, Pierce T. O'Neil ${ }^{1}$, Bradley L. Pentelute ${ }^{2}$, Maria T. Villar ${ }^{1}$, Antonio Artigues ${ }^{1}$, Mark T. Fisher ${ }^{1}$ \\ ${ }^{1}$ Department of Biochemistry and Molecular Biology, University of Kansas Medical Center \\ ${ }^{2}$ Department of Chemistry, Massachusetts Institute of Technology
}

Correspondence to: Mark T. Fisher at mfisher1@kumc.edu

URL: https://www.jove.com/video/57902

DOI: doi:10.3791/57902

Keywords: Biochemistry, Issue 138, Biolayer interferometry, electron microscopy, mass spectrometry, anthrax toxin, protein complexes, biosensor

Date Published: 8/6/2018

Citation: Machen, A.J., O'Neil, P.T., Pentelute, B.L., Villar, M.T., Artigues, A., Fisher, M.T. Analyzing Dynamic Protein Complexes Assembled On and Released From Biolayer Interferometry Biosensor Using Mass Spectrometry and Electron Microscopy. J. Vis. Exp. (138), e57902, doi:10.3791/57902 (2018).

\section{Abstract}

In vivo, proteins are often part of large macromolecular complexes where binding specificity and dynamics ultimately dictate functional outputs. In this work, the pre-endosomal anthrax toxin is assembled and transitioned into the endosomal complex. First, the $\mathrm{N}$-terminal domain of a cysteine mutant lethal factor $\left(\mathrm{LF}_{\mathrm{N}}\right)$ is attached to a biolayer interferometry $(\mathrm{BLI})$ biosensor through disulfide coupling in an optimal orientation, allowing protective antigen (PA) prepore to bind $\left(K_{d} 1 \mathrm{nM}\right)$. The optimally oriented $L_{\mathrm{N}}-\mathrm{PA}_{\text {prepore }}$ complex then binds to soluble capillary morphogenic gene-2 (CMG2) cell surface receptor $\left(\mathrm{K}_{d} 170 \mathrm{pM}\right)$, resulting in a representative anthrax pre-endosomal complex, stable at $\mathrm{pH} 7.5$. This assembled complex is then subjected to acidification $\left(\mathrm{pH} \mathrm{5.0)}\right.$ representative of the late endosome environment to transition the $\mathrm{PA}_{\text {prepore }}$ into the membrane inserted pore state. This $\mathrm{PA}_{\text {pore }}$ state results in a weakened binding between the CMG2 receptor and the $L F_{N}-P A_{\text {pore }}$ and a substantial dissociation of $C M G 2$ from the transition pore. The thio-attachment of $L_{N}$ to the biosensor surface is easily reversed by dithiothreitol. Reduction on the $B L I$ biosensor surface releases the $L_{N}-P A_{\text {prepore }}-C M G 2$ ternary complex or the acid transitioned $L F_{N}-P A_{\text {pore }}$ complexes into microliter volumes. Released complexes are then visualized and identified using electron microscopy and mass spectrometry. These experiments demonstrate how to monitor the kinetic assembly/disassembly of specific protein complexes using label-free BLI methodologies and evaluate the structure and identity of these BLI assembled complexes by electron microscopy and mass spectrometry, respectively, using easy-to-replicate sequential procedures.

\section{Video Link}

The video component of this article can be found at https://www.jove.com/video/57902/

\section{Introduction}

Identifying and understanding the specificity governing protein complex assembly in vivo is of extreme interest for biochemical researchers. Large heterogeneous protein assemblies are the norm rather than the exception. This notion is supported by spectroscopic monitoring of in vivo assembly, isolating complexes using gentler cell disruption techniques, evaluating products from affinity-based purification methods, and visualizing them using high resolution cryogenic electron microscopy (cryo-EM). To understand the control of assembly specificity within the cell, researchers must routinely isolate, identify, and ultimately characterize these dynamic assembling/disassembling structures. The most heavily used molecular tool to identify the components of these assemblies frequently requires antibody-based immunoprecipitation, which relies on maintaining complex stability during cell disruption. Various coupled analytical techniques were recently developed to capture complexes from cell samples using microfluidic based approaches, such as surface plasmon resonance (SPR). Following removal from the SPR surfaces, these samples were analyzed by matrix-assisted laser desorption/ionization time of flight (MALDI-TOF) ${ }^{1,2}$. Advancing this methodology using easier protocols will allow researchers to visualize and validate predicted complexes that occur in the cellular milieu. Since SPR is a microfluidics-based system, problems often arise from aggregate formation. Circumventing this problem requires sample dilution, which in turn, can decrease the integrity of concentration liable biological complexes.

A relatively recent advancement in label-free technologies is the development of the biolayer interferometry (BLI) system ${ }^{3}$. These particular lightbased reflectance systems replicate, or best emulate, SPR binding and kinetic results at a fraction of the cost ${ }^{3,4}$ particularly if single channel units are used. BLI measures changes in reflected light interference patterns between a reference layer (control) and the biolayer surface (experimental). The resulting change in phase is measured in real time as a kinetic and quantitative readout ${ }^{5}$. The biosensor surface, containing specific immobilization chemistries, is physically transferred between solutions, as opposed to buffer changes by microfluidic approaches in SPR, for measurement via wavelength phase deflections. Mass transfer effects are prevented by agitating the solution. Unlike SPR, these systems are quite useful in evaluating complexes from crude biological samples. The physical parameter measured during BLI experiments primarily depends on a change in mass or thickness at the biosensor surface which results from protein complex assembly or disassembly. 
These fiber optic biosensors are easy to use and relatively inexpensive. One of the emerging useful aspects of BLI is the facile removal of newly assembled protein complexes from the surface. A recent application of this method allowed our laboratory to observe the real time kinetics of large scale $\mathrm{pH}$ induced protein structure rearrangement of the anthrax toxin protective antigen (PA) component as the prepore (PA $\mathrm{prepore})$ transitioned to its pore $\left(\mathrm{PA}_{\text {pore }}\right)$ form. This transition on the biosensor tip was verified using electron microscopy $(\mathrm{EM})^{6}$. Removal of complexes from biosensor surfaces avoids larger volume dilution effects frequently encountered when releasing complexes from chip surfaces when using microfluidics systems.

In the current work, anthrax toxin complexes are assembled and disassembled on the biosensor surface and then released into microliter volumes. The resulting complex components are validated orthogonally using EM and mass spectrometry (MS).

\section{Protocol}

\section{Assembly of Defined Macromolecular Complexes on Biolayer Interferometry (BLI) Biosensor Surfaces}

1. Assembly of prepore anthrax toxin complex on a PDEA-modified amine reactive biosensor surface

1. Hydrate an amine reactive second generation (AR2G) BLI biosensor tip in $250 \mu \mathrm{L}$ of water for ten minutes.

2. Program step times, listed in Table 1, on the software controlling the BLI unit (see Table of Materials).

3. Start the BLI run by immersing the biosensor tip in $250 \mu \mathrm{L}$ water for $30 \mathrm{~s}$ to measure an initial baseline of biosensor thickness and density.

4. Activate the biosensor by immersing the tip into $250 \mu \mathrm{L} 50 \mathrm{mM} \mathrm{NHS}$ (N-hydroxysuccinimide) and $200 \mathrm{mM}$ EDC (1-ethyl-3-(3dimethylaminopropyl)carbodiimide)) for 7 minutes.

5. Immerse the activated biosensor in $50 \mathrm{mM}$ PDEA (2-(2-pyridinyldithio)ethanamine) dissolved in $0.1 \mathrm{M}$ borate buffer ( $\mathrm{pH} 8.5$ ) for 5 minutes to generate an activated thiol-reactive surface.

6. Immerse the activated thiol-reactive biosensor into $250 \mu \mathrm{L}$ of solution containing $100 \mathrm{nM} \mathrm{E126C} \mathrm{LF} \mathrm{N}_{\mathrm{N}}$ in $10 \mathrm{mM}$ sodium acetate pH 5.0 , $100 \mathrm{mM} \mathrm{NaCl}$, buffer for 5 minutes.

7. Immerse the $\mathrm{LF}_{\mathrm{N}}$ tip in $50 \mathrm{mM} \mathrm{L}$-cysteine, $1 \mathrm{M} \mathrm{NaCl}, 0.1 \mathrm{M}$ sodium acetate, $\mathrm{pH} 5.0$, for 4 minutes, to quench any remaining reactive free thiol-reactive groups.

8. Immerse the quenched $\mathrm{LF}_{\mathrm{N}}$ tip in $50 \mathrm{mM}$ Tris, $50 \mathrm{mM} \mathrm{NaCl}$ for 2 minutes to establish buffer baseline

9. Immerse the quenched $\mathrm{LF}_{\mathrm{N}}$ tip into $0.5 \mu \mathrm{M}$ protective antigen prepore ( $\left(\mathrm{A}_{\text {prepore }}\right), 50 \mathrm{mM}$ Tris, $50 \mathrm{mM} \mathrm{NaCl}$ for 5 minutes to create the $\mathrm{LF}_{\mathrm{N}}-\mathrm{PA} \mathrm{p}_{\text {prepore }}$ complex.

10. Once $P A_{\text {prepore }}$ is associated, remove the tip from the $P A_{\text {prepore }}$ solution and immerse the tip into $50 \mathrm{mM}$ Tris, $50 \mathrm{mM} \mathrm{NaCl}$ for 30 seconds to wash away any non-specifically bound $P A_{\text {prepore. }}$.

11. Immerse the $\mathrm{LF}_{\mathrm{N}}-\mathrm{PA}_{\text {prepore }}$ complex into $0.5 \mu \mathrm{M}$ CMG2 receptor (without the transmembrane domain), $50 \mathrm{mM} \mathrm{Tris,} 50 \mathrm{mM} \mathrm{NaCl}$, for 5 minutes.

12. Immerse the $\mathrm{LF}_{\mathrm{N}}-\mathrm{PA}$ prepore-CMG2 complex into $50 \mathrm{mM}$ Tris, $50 \mathrm{mM} \mathrm{NaCl}$ for 30 seconds to wash away any unbound CMG2 to form preendosomal complex.

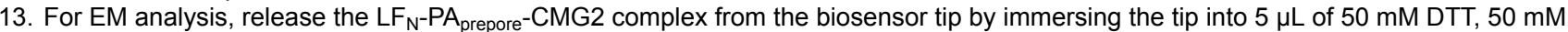
Tris, $50 \mathrm{mM} \mathrm{NaCl}$, inside a PCR tube.

14. For tandem MS analysis of the peptides from the complex, release the $\mathrm{LF}_{\mathrm{N}}-\mathrm{PA}_{\text {prepore }}-\mathrm{CMG} 2$ complex from the biosensor tip by immersing the biosensor into $5 \mu \mathrm{L} 50 \mathrm{mM}$ DTT, $6 \mathrm{M} \mathrm{GuHCl}$ (keratin-free), $25 \mathrm{mM}$ ammonium bicarbonate pH 8.0 inside a PCR tube. This is performed on a different biosensor than the one used for EM analysis.

2. Assembly of pore anthrax toxin complex on PDEA-modified amine reactive biosensor surface

1. To view the complex after $\mathrm{pH}$ transition, perform steps 1.1.1-1.1.12 in the previous section to generate the $\mathrm{LF}_{\mathrm{N}}-\mathrm{PA} \mathrm{A}_{\text {prepore }}-\mathrm{CMG} 2$ complex.

2. Immerse the biosensor tip into a $10 \mathrm{mM}$ acetate $\mathrm{pH} 5.0$ for 5 minutes to initiate the transition of the $\mathrm{PA}_{\text {prepore }}$ to $\mathrm{PA}_{\text {pore }}$ transition. This transition is indicated by increasing amplitude (approximately $0.2 \mathrm{~nm}$ ) followed by a larger amplitude decline that is hypothesized to be substantial or complete receptor dissociation due to diminished binding affinity.

3. Immerse $\mathrm{LF}_{\mathrm{N}}-\mathrm{PA}$ pore tip in $50 \mathrm{mM}$ Tris, $50 \mathrm{mM} \mathrm{NaCl}, \mathrm{pH}$ 8.0, for 30 seconds to wash off acidic buffer.

4. For the EM analysis sample, immerse the biosensor tip into a solution containing $1.25 \mathrm{mM}$ micelles (2.5 mM MSP1D1, 25 mM Nacholate, $162.5 \mathrm{mM}$ POPC) for 5 minutes to prevent aggregation in solution after disulfide release.

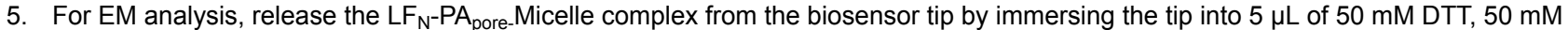
Tris, $50 \mathrm{mM} \mathrm{NaCl}$ inside a PCR tube.

6. For tandem MS analysis of the peptides from the complex, release the $\mathrm{LF}_{\mathrm{N}}-\mathrm{PA}_{\text {poore }}$ complex (no micelle) from the biosensor tip by immersing the biosensor into $5 \mu \mathrm{L} 50 \mathrm{mM}$ DTT, $6 \mathrm{M} \mathrm{GuHCl}$ (keratin-free), $25 \mathrm{mM}$ ammonium bicarbonate pH 8.0 inside a PCR tube. This is performed on a different biosensor than the one used for EM analysis.

\section{Visualizing and Validating Released Macromolecular Assemblies from BLI Biosensors by Negative Stain Electron Microscopy}

1. Glow discharge a carbon-coated $\mathrm{Cu} 300$ grid. Typical glow discharge settings are $0.38 \mathrm{mBar}$ stable atmosphere pressure, negative 15 mAmps, 20 seconds, then venting with air.

2. Secure grid between a pair of clean tweezers.

3. Pipette $4 \mu \mathrm{L}$ of released complex sample in PCR tube onto the grid and allow adsorption for $60 \mathrm{~s}$.

4. Wick away remaining liquid with a filter paper wedge. 
5. Stain the grid by pipetting $5 \mu \mathrm{L}$ of $0.75 \% 0.02$ micron filtered uranyl formate and wicking away excess stain after 5 seconds. Allow grid to dry at room temperature.

6. View stained sample grids using a transmission electron microscope.

\section{Identification of Complete Pre-endosomal Anthrax Toxin Complex ( $\left.\mathrm{LF}_{\mathrm{N}}-\mathrm{Pa}_{\text {prepore}}-\mathrm{CMG}\right)$ and Transitioned Complex $\left(\mathrm{LF}_{\mathrm{N}}-\mathrm{Pa}_{\text {pore }}\right.$ without $\left.\mathrm{CMG}\right)$ Using Mass Spectrometry.}

1. Dilute released samples to $20 \mu \mathrm{L}$ and incubate for 1 hour.

2. Add $2 \mu \mathrm{L}$ of $55 \mathrm{mM}$ iodoacetamide, $25 \mathrm{mM}$ ammonium bicarbonate, $\mathrm{pH} 8.0$, and incubate for 1 hour at room temperature in dark (covered with aluminum foil).

3. Dilute the sample with $100 \mu \mathrm{L}$ of $25 \mathrm{mM}$ ammonium bicarbonate, $\mathrm{pH} 8.0$, to reduce the guanidine hydrochloride concentration below $1 \mathrm{M}$.

4. Add $5 \mu \mathrm{L}$ of sequencing grade modified trypsin at $20 \mathrm{ng} / \mu \mathrm{L}$ and incubate at $37^{\circ} \mathrm{C}$ overnight.

5. Add glacial acetic acid to a final concentration of $5 \%$ to reduce the $\mathrm{pH}$ to $<3$, then reduce the volume to $10 \mu \mathrm{L}$ in vacuum concentrator.

6. Transfer the peptide solution to the sample plate on the autosampler of the nLC 1200 uHPLC.

7. Load $5 \mu \mathrm{L}$ the peptide solution onto a uHPLC reversed phase column mounted on the ionization stage of the MS.

8. Wash column with $15 \mu \mathrm{L}$ of $0.1 \%$ formic acid at a maximum rate of $5 \mu \mathrm{L} / \mathrm{min}$ and/or maximum pressure of $800 \mathrm{psi}$.

9. Elute peptides from the reversed-phase $\mathrm{C} 18$ column at a flow rate of $350 \mathrm{~nL} / \mathrm{min}$ over a $90 \mathrm{~min}$ period using a lineal gradient of $5 \%$ to $40 \%$ of solvent $B$ in $A+B$ (solvent $A: 0.1 \%$ formic acid; solvent $B$ : $95 \%$ acetonitrile with $0.1 \%$ formic acid).

10. Analyze eluting peptides on line using tandem MS.

1. Set the ionization source to 2500 volts and ion transfer temperature to $250{ }^{\circ} \mathrm{C}$.

2. Operate the mass spectrometer under automatic control to perform continuously one MS scan followed by as many tandem MSMS as possible in a 3 s period, using CID and a normalized collision energy of 35.

11. Identify peptide and protein components using standard methods. For this work, two sets of peptide and protein analysis were performed.

\section{Representative Results}

The ability to monitor and validate the assembly of large macromolecular complexes is a crucial step towards understanding the specificity and functionality of large biomolecular assemblies. The results of the methods presented herein demonstrate the ease with which large protein complexes (>150 kDa mass) can be assembled using biolayer interferometry, all while monitoring the kinetics and amplitude of assembly. The unique compact nature of the biosensor surface enables assembly analysis to be extended by releasing assembled complexes into small microvolumes. These microvolumes can be used to visualize the initial physical structure of the complexes using EM and to verify the identity of the complex components using MS. A schematic overview of this entire process is shown in Figure 1.

A key element for the successful assembly and verification of macromolecular complex on the biosensor surface involves the proper orientation of the initial seed protein. This ensures that the protein-protein interaction sites are accessible, not sterically blocked, and optimally positioned away from the biosensor surface. As shown in Figure 2, the proper orientation of the anthrax toxin complex is achieved by using a specifically engineered $\mathrm{N}$-terminal fragment of lethal factor $\left(L F_{N}\right)$ so the $L F_{N}-P A_{\text {prepore }}$ binding site is always positioned opposite of the biosensor covalent attachment site ${ }^{6,7}$. The subsequent buildup of the complex with binding of PA to the LF $F_{N}$ BLI biosensor followed by soluble CMG2 binding to PA ultimately creates a translocation competent anthrax toxin complex.

The BLI sensogram trace is a real-time read out of the amplitude changes due to specific addition of the anthrax toxin components as they are added onto the biosensor. Figure 3 shows a representative trace paired with a model of the complex predicted to form at that step in the process. The first rise is $L F_{N}$ loading onto the tip. After quenching and baseline, $P A_{\text {prepore }}$ then binds to $L F_{N}$ followed by the addition of soluble CMG2 receptor resulting in the assembled pre-endosomal complex of $L F_{N}-P A_{\text {prepore }}-C M G 2$. To progress toward the late endosome environment, the entire complex is subjected to a low $\mathrm{pH}$ pulse $(\mathrm{pH}$ 5.0) that weakens the receptor binding, allowing the prepore to transition to its extended membrane inserted pore conformation. ${ }^{6}$ Sensogram traces of the acidification step are shown in Figure 4. The initial increase or 'spike' in amplitude is likely the pore extension ${ }^{6}$ that must occur prior to the decrease in the CMG2 receptor binding. The larger amplitude decline is most likely substantial or complete receptor dissociation due to diminished binding affinity. Previous work in this laboratory indicated CMG2 binding to the fully extended $\mathrm{PA}_{\text {pore }}$ is negligible compared to the CMG2-PA $\mathrm{Arepore}_{\text {interaction }}$. In addition, the sensogram kinetic trace, observed in all sensograms of $L F_{N}-P A_{\text {prepore }}-C M G 2$ transitions to $L F_{N}-P A_{\text {pore }}$ with a pH drop, is reproducible over multiple runs.

Prior to and after acidification, the biosensor attached complexes are easily released for visualization by negative stain EM and identification by MS (Figure 5). Representative complexes from the EM results are shown in Figure 6. Pre-endosomal sample grids, show densities consistent with intact ternary complexes consisting of $\mathrm{LF}_{\mathrm{N}}-\mathrm{PA}_{\text {prepore}}-\mathrm{CMG}$. Post-acidification complex grids, show PA transitioned to pore and solubilized by micelle inclusion with no obvious CMG2 density.

The identities of the pre- and post-acidification complexes were verified by MS. A database where the sequences of PA, P13423; LF ${ }_{N}, P 15917$; and CMG2, P58335 were included in a background of a mouse proteins database derived from the NCBInr repository. Only the proteins of interest were obtained from this first database search, with the following amino acid coverage for the ternary and binary complexes: $54 \%$ and $22 \%$ for PA, $36 \%$ and $6 \%$ for LF and $43 \%$ for CMG2, respectively (CMG2 was not detected on the binary complex). In order to maximize the protein amino acid coverage, a second peptide/protein identification was performed using a protein database containing only the three proteins of interest. Pre-endosomal MS samples contained peptides from all three toxin components with $60.46 \%, 67.97 \%$, and $54.15 \%$ coverage for $\mathrm{LF}_{\mathrm{N}}, \mathrm{PA}$, and CMG2, respectively (Figure 7). Post-endosomal results, shown in Figure 8, only contained peptides from LF $\mathrm{F}_{\mathrm{N}}$ and PA $(57.41 \%$ and $67.79 \%$ coverage, respectively). The lack of CMG2 in the post-endosomal samples are consistent with the observed BLI nm decrease during pore formation. 


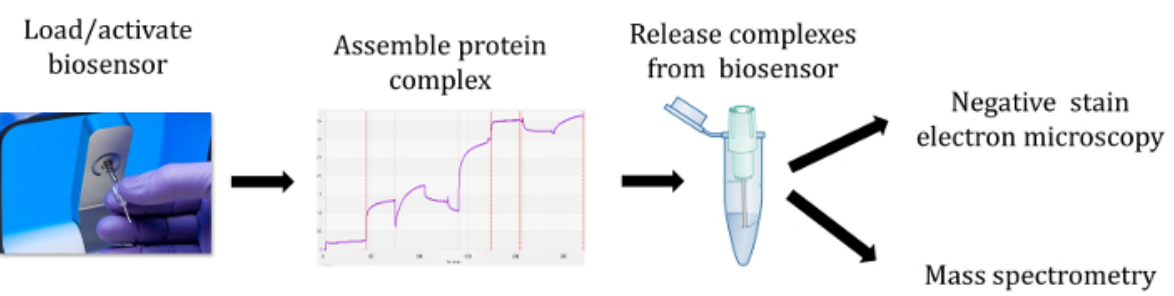

Figure 1. Schematic overview for analysis of protein complexes assembled on and isolated from BLI biosensor using EM and MS. Please click here to view a larger version of this figure.

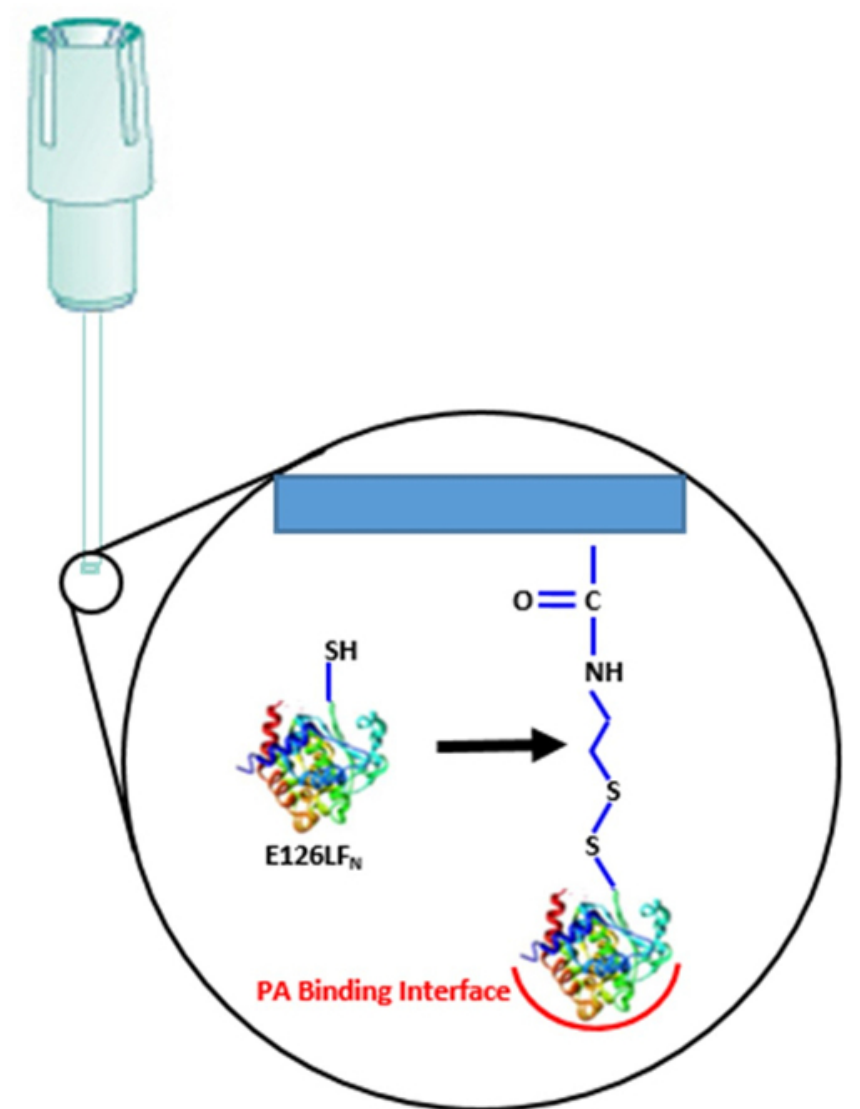

Figure 2. Biosensor activation: First step in orientation specific assembly on BLI biosensor. The E126C Lethal Factor $N$-terminal domain, $\left(\mathrm{LF}_{\mathrm{N}}\right)$ is linked through a thio-linkage creating the properly orientated $\mathrm{PA}_{\text {prepore }}$ binding interface. Please click here to view a larger version of this figure. 


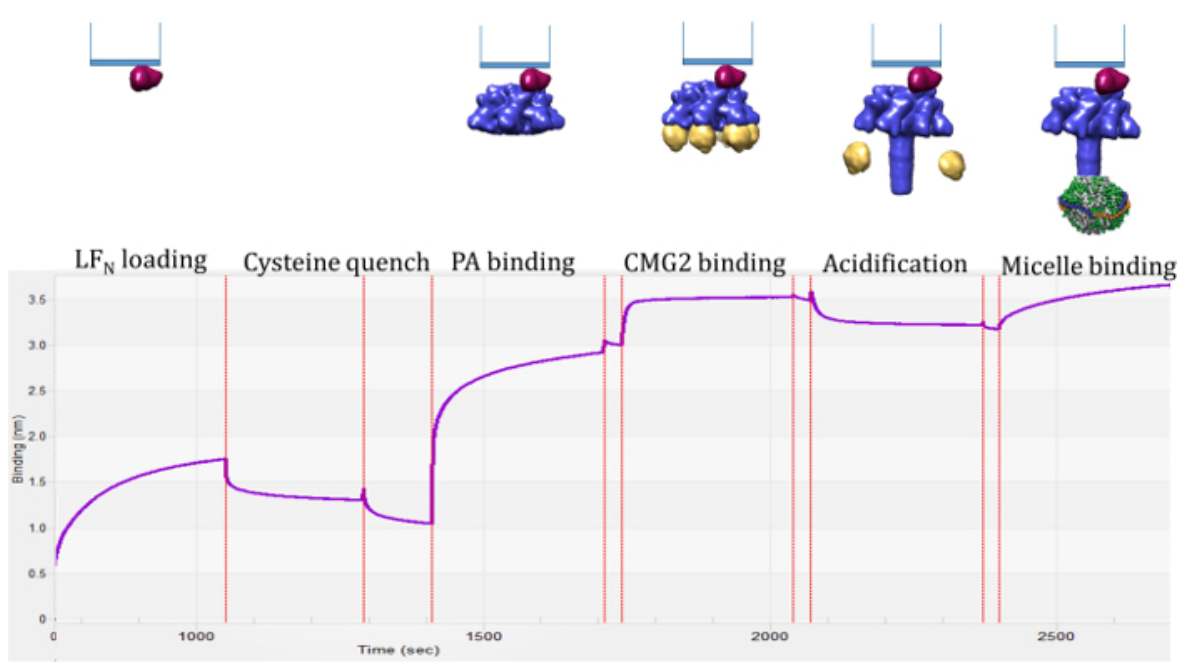

Figure 3. Monitoring anthrax toxin assembly and disassembly with BLI: The sensogram shows amplitude changes as a function of time due to specific addition of the anthrax toxin components as they are added onto the biosensor starting with the LF $_{N}$ loading (step 4 in Table 1). PA $A_{\text {prepore }}$ is then loaded onto the surface followed by the addition of soluble CMG2 receptor. The assembled pre-endosomal complex consists of an LF$\mathrm{PA}_{\text {prepore}}-\mathrm{CMG}$. To progress toward the late endosome environment, the entire assemble functional anthrax toxin is subjected to a low pH pulse ( $\mathrm{pH} 5.0)$ that weakens the receptor binding, allowing the prepore to transition to its extended membrane inserted pore conformation. Exposure of the membrane spanning pore is confirmed and solubilized by addition of micelles. Please click here to view a larger version of this figure.

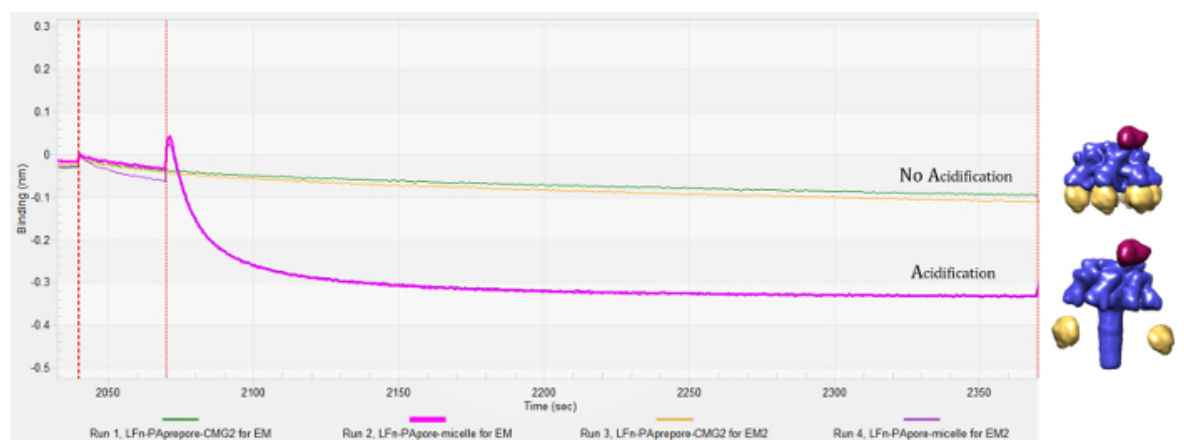

Figure 4. BLI sensogram of CMG2 release: Traces of the acidification step show an initial increase or 'spike' in amplitude followed by a larger amplitude decline that are likely pore formation and receptor dissociation, respectively. Vertical dotted red lines denote start of a new step. Sensograms are aligned at the bolded dotted red line. Please click here to view a larger version of this figure.

Release complexes from biosensor

Electron microscopy

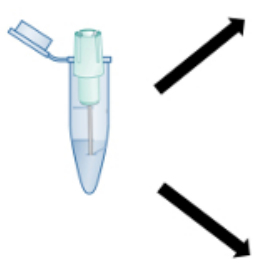

Mass spectrometry
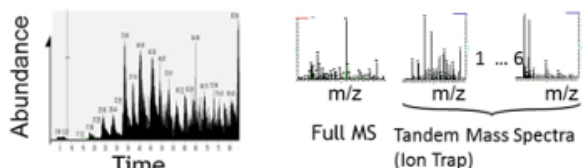
Full MS Tandem Mass Spectra
(Ion Trap)

Figure 5. Analyzing protein complexes assembled on and isolated from BLI biosensor using EM and MS: Biosensor attached complexes are easily released into $5 \mu \mathrm{L}$ of buffer containing DTT for visualization by negative stain EM and identification by MS. Please click here to view a larger version of this figure. 


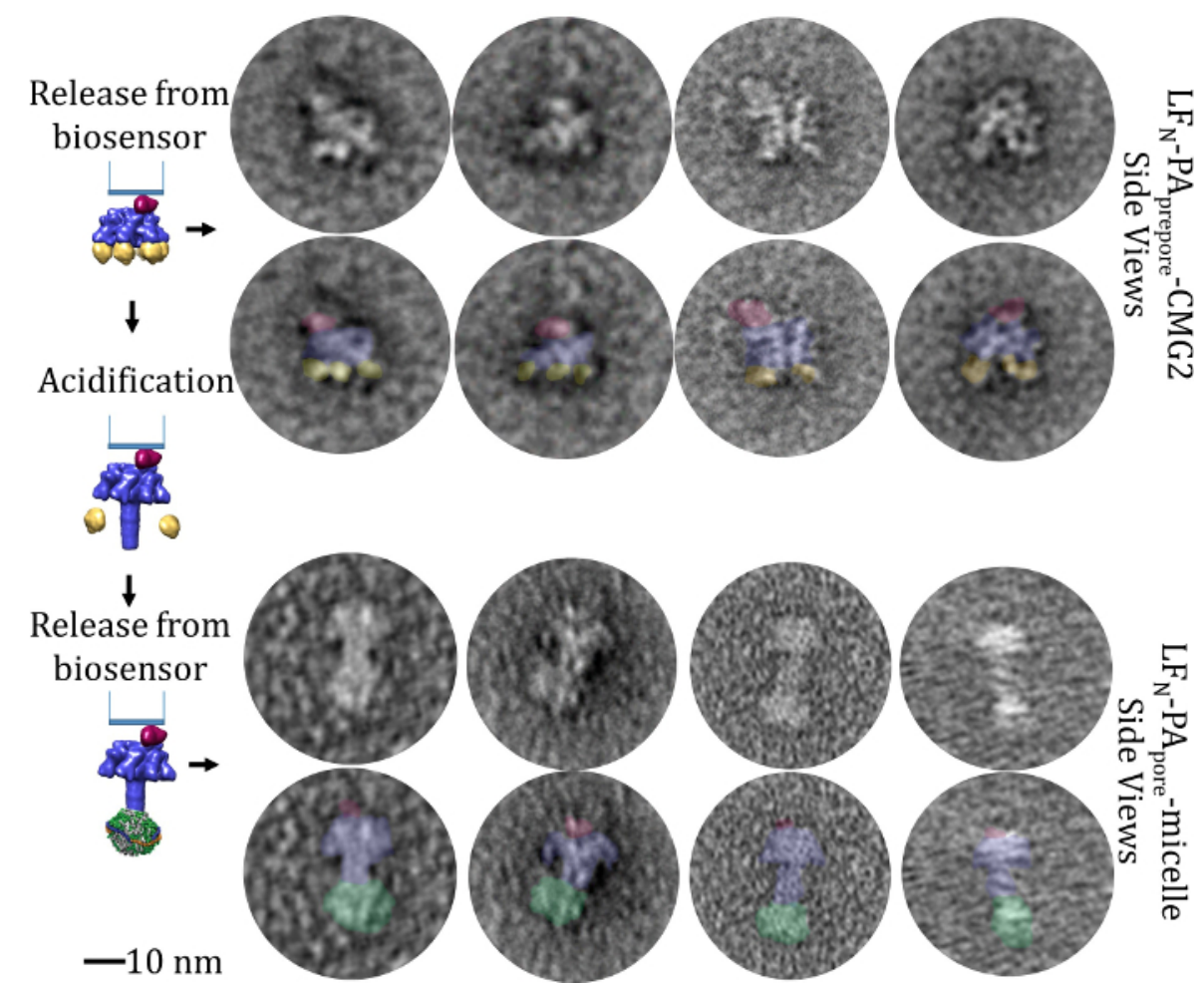

Figure 6. Visualization of protein complexes with EM: Pre-endosomal sample grids, show densities consistent with intact ternary complexes consisting of $\mathrm{LF}_{\mathrm{N}}-\mathrm{PA} \mathrm{A}_{\text {prepore}}-\mathrm{CMG}$. Post-endosomal complex grids, show PA transitioned to pore and solubilized by micelle with no obvious CMG2 density. Models of predicted complexes (left hand side) are at the same scale as individual particles shown. Particles colorized with predicted protein (based on size of EM density) are shown below each particle. Please click here to view a larger version of this figure.

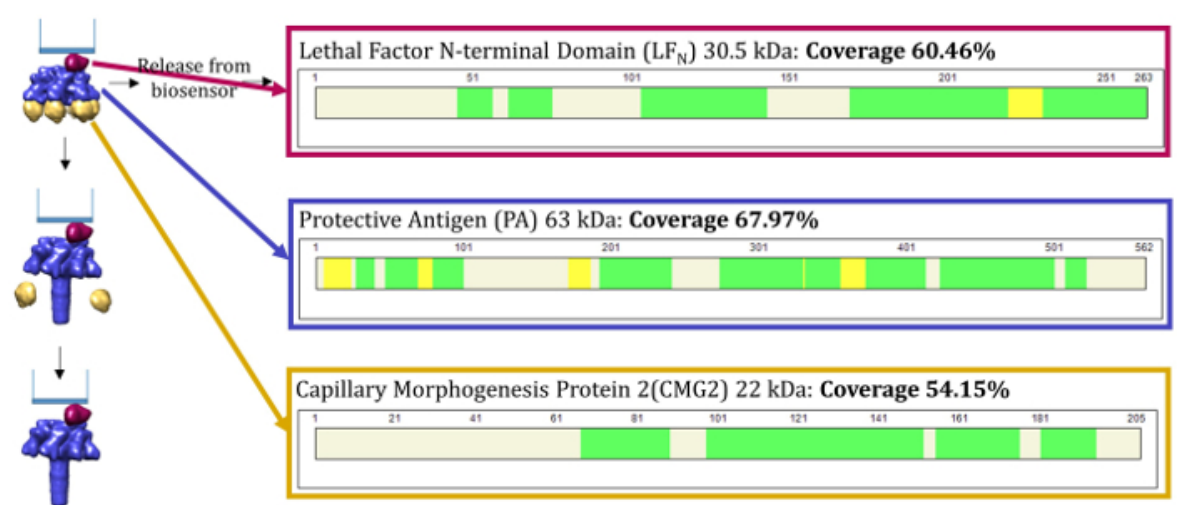

Figure 7. Verification of protein complexes with MS: Pre-endosomal MS samples contained peptides from all three toxin components with $60.46 \%, 67.97 \%$, and $54.15 \%$ coverage for $\mathrm{LF}_{\mathrm{N}}, \mathrm{PA}$, and $\mathrm{CMG}$, respectively. Peptides detected at a false discovery rate (FDR) equal or higher than $5 \%$ shown in yellow, FDR equal or higher than $1 \%$ shown in green. Please click here to view a larger version of this figure. 


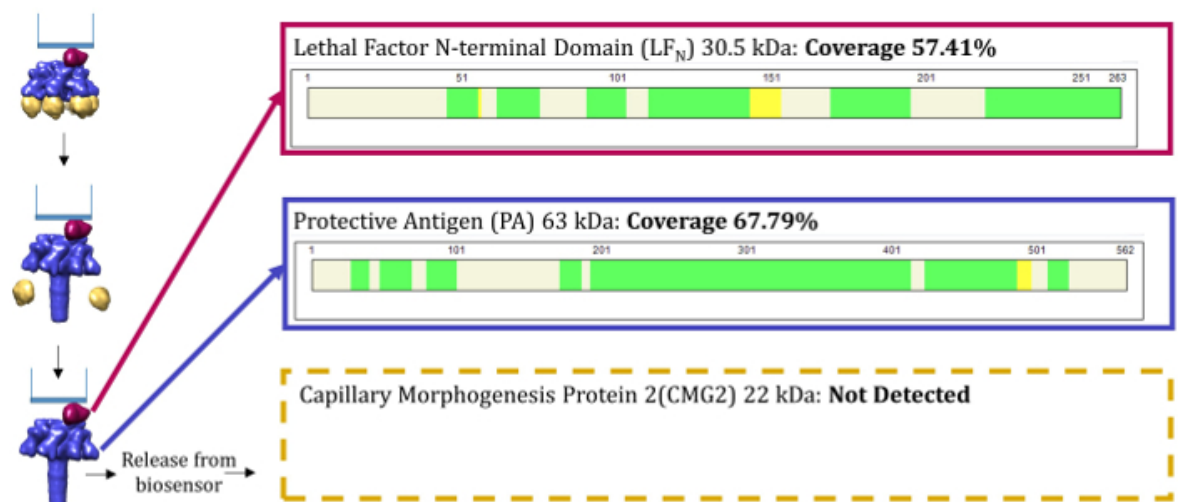

Figure 8. Verification of protein complexes with MS: Post-endosomal samples contained peptides from $\mathrm{LF}_{\mathrm{N}}$ and $\mathrm{PA}(57.41 \%$ and $67.79 \%$ coverage, respectively), but not CMG2. FDR equal or higher than $5 \%$ shown in yellow, FDR equal or higher than $1 \%$ shown in green. Please click here to view a larger version of this figure.

\begin{tabular}{|l|l|l|}
\hline Step & Time (s) & Description \\
\hline 1 & 30 & Initial baseline \\
\hline 2 & 420 & Activation \\
\hline 3 & 300 & Activation \\
\hline 4 & 300 & LF $_{\mathrm{N}}$ loading \\
\hline 5 & 240 & Cysteine quench \\
\hline 6 & 120 & Baseline \\
\hline 7 & 300 & PA prepore $_{\text {association }}$ \\
\hline 8 & 30 & Baseline \\
\hline 9 & 300 & CMG2 association \\
\hline 10 & 30 & Baseline \\
\hline 11 & 300 & Acid transition \\
\hline 12 & 30 & Baseline \\
\hline 13 & 300 & Micelle association \\
\hline
\end{tabular}

Table 1. Step times for single BLI run to assembly anthrax toxin complex. Note that baselines are used to wash away unbound protein from previous step and/or establish a new buffer baseline.

\section{Discussion}

This demonstration illustrates how macromolecular complex formation can be easily monitored using biolayer interferometry, visualized using EM, and verified with MS, all with microvolumes in a short time span. Structural assembly and observed complexes follow the biologically relevant predictions, further validating this combined methodology. As mentioned in the results section, the key element for assembly success requires rationally engineered cysteine mutagenesis to ensure that the complex protein-protein interfaces are properly oriented away from the biosensor surface.

Previous systems have used BLI and MS techniques to evaluate protein binding of two and three component systems as well as integrity of receptor binding of expressed protein, but, in both instances, the methods were not developed to take advantage of the tandem EM/ MS approach ${ }^{8,9}$. The only other interferometry system that combined MS analysis to help characterize interactions was a dual-polarization interferometry ${ }^{10}$. Unfortunately, this system is no longer available for general use. As mentioned in the introduction, there have been a number of studies completed where samples were formed on SPR-like biosensor surfaces and removed from MS analysis. None of those examples resulted in complexes visualized using EM.

The limitations of this sequential method can be numerous but are solvable. For the initial immobilization and therefore foundation step of the assembly, a lack of knowledge of the structural interaction surfaces would certainly impede progress associated with monitoring initial assembly phases. The lack of structure information can be addressed by designing an engineered foundation construct where attachment chemistries (e.g. sulfhydryl moiety for disulfide linkages/ His-tagged positioning) can be moved to various regions within the core assembly system. In the case of the anthrax toxin complex, it was fortunate that the structure of $\mathrm{LF}_{\mathrm{N}}$ bound to $\mathrm{PA}_{\text {prepore }}$ is available ${ }^{11}$. Rational placement of the engineered cysteine was localized to regions away from the $\mathrm{PA}_{\text {prepore }}$ binding face. With cysteine linkage chemistries, it is preferable that no other reactive cysteines are present on the protein surface.

There are various attachment chemistries which can be used to engineer specific attachment site on a protein's surfaces. One of the most popular specific attachments involves positioning a biotin moiety at a specifically defined location on the protein surface ${ }^{12}$. Unfortunately, biotin 
binding to a streptavidin or avidin coated surfaces is quite tight. Reversal of the binding interaction is not simple. The use of an engineered Histag at the $\mathrm{N}$ - or C-terminus and the subsequent ease of attachment to Ni-NTA surfaces is a more universal application of affinity immobilization. Of course, one of the caveats for engineering assembly attachment sites with His-tagged systems is the requirement that the $\mathrm{N}$ - and $\mathrm{C}$-termini of the core assembly protein remain exposed and separated so that the attachment is easy. As with all assembly processes, the interaction interface of the core assembly protein must remain available as the complex assembly progresses.

Perhaps the most common concern of using biosensor surface chemistries is non-specific binding. Streptavidin tips are often a source of significant non-specific binding effects. Disulfide linked biotin can be used to release very specific complexes leaving behind the reduced $\mathrm{S}$-biotin linkage tightly bound to immobilized streptavidin biosensors ${ }^{13}$. There are other reversible chemistries becoming available such as iminoboronates and to a lesser extent ketoamide ${ }^{14}$. This field is currently underdeveloped, but there is high interest in further developing reversible covalent protocols to avoid off-target drug toxicity effects that commonly accompany the use of covalent targeted drug development.

A limitation of using EM to visualize complexes is interpretation, especially in instances where the structures of the assembled complexes are not initially known. The spatial location of components within an undefined assembled macromolecular complex can be identified using monoclonal antibodies $(\mathrm{mAb})$ as specific kinetic and structural markers. For example, once a complex is formed, monoclonal antibodies can be added that bind to specific components. This method is frequently used in EM to identify specific components within large assemblies ${ }^{15}$. Another limitation is related to the size of the complex, although there have been instances where defined symmetric assemblies as small as $70 \mathrm{kDa}(\mathrm{GroES}$ heptamer) are easily resolved using negative stain EM. Assembled complexes that are analyzed by EM are typically in the size range of $\sim 100$ $\AA$ in diameter or above. Recently however, proteins as small as $20 \mathrm{kDa}$ have been resolved and low resolution structures have been obtained when using superior staining methodologies ${ }^{16}$.

For MS analysis, the increased sensitivity of current MS instrumentation down to the femtomolar (attomoles) level can in some cases increase the sensitivity of BLI detection. It is highly conceivable that protein signals that show a minimal but repeatable rise in amplitudes will result in identification of the protein in question. In addition, probing protein-protein interactions with one of the partners attached on the biosensor and the other in a cellular milieu will in effect result in a purification and subsequently easier detection of the newly formed complex. One limitation that may be observed with the current highly sensitive MS systems is that the protein of interest may not be in the database, but this observation is rare (e.g., proteomes from rare species). If the sequences of the proteins of interest are known, this problem is easily solved by including the protein(s) amino acid sequence in a background protein database (as described in this work in the protocol section). Another potential limitation of the methodology results from the resistance of a protein to trypsinolysis. Trypsin digestion is typically the default method for bottom up protein identification. However, proteins may be resistant to trypsin if they lack Arg and Lys residues or access to these residues are restricted by the folded structure. These limitations are resolved, respectively, by using alternative or a combination of proteases or including an unfolding reagent (urea or guanidine $\mathrm{HCl}$, as indicated in 1.1.14 and 1.2.6) before enzymatic digestion.

Possible expansions of this methodology include allowing the user to follow and identify cellular assembly complexes from crude cellular lysates. It turns out testing for assembly components in concentrated cellular extracts is easy to perform using the biolayer interferometry procedures. Unlike more commonly used microfluidic based methodologies which are prone to clogging and sensitive to aggregation, the BLI approach can be used to directly immerse biolayer sensor tips into crude extracts to potentially assemble specific complexes directly from these concentrated impure samples. Once assembled, it is entirely feasible to use specific antibody probes as a follow up to the BLI system to further identify and even quantitate suspected components in cellular extracts that were identified using the microvolume MS method. Again, the key here is to use defined, properly oriented core proteins as specific affinity probes.

The ability to view the prepore to pore transition process kinetically with BLI will be highly useful in identifying potential "anti-toxin" small molecule inhibitors of the protein transitions that specifically function under late endosomal, low pH (5.0) conditions. This pH induced prepore to pore transition is inhibited in the presence of folding stabilizer (osmolytes) such as glycerol or sucrose, and thus lends strong support for developing specific targeted folding stabilizers that prevent $\mathrm{PA}_{\text {pore }}$ formation. This specific approach avoids and replaces crude aggregation-based assays where $\mathrm{pH}$ drops lead to protein precipitation. This latter method, although good for primary prescreening methods, often leads to false positive results where specific compounds inhibit the aggregation rather than the actual molecular transitions.

The downstream observations of the structure and identification of the individual assembled components within small microvolume samples can also be useful in validating potential lead compounds. This can be applied in instances where either specific assembly stabilization or destabilization is the target outcome. This kinetic/structure/identification parallel approach is useful for directly confirming the validity of suspected lead compound effectors of assembly and serves as a reasonable secondary screening step or medium throughput approach.

Cryo-EM is a useful technique to study the atomic details of macromolecule complexes in various states of assembly. Prior to preparing cryoEM samples, it is important to first verify a preparation contains reasonably pure homogenous complexes with negative stain EM. The work presented herein demonstrates assembly of protein complexes on BLI biosensor surfaces, release of these complexes for EM visualization, and identification of these components using MS. This particular methodology of controlled assembly and release can be useful in generating very specific protocols that enhance homogenous sequential sample preparation for negative stain EM, a necessary step that must be demonstrated before advancing to cryo-EM. To obtain low resolution 3D structure, only 30-50 particles of complex would be needed to perform a conical tilt series (70 different 2D image views per particle) provided there is orientation diversity (multiple different views).

With respect to enhancing MS methods, advances in sensitivity and reduction in sample volume continue to improve. Nano flows and ultra-high pressure liquid chromatography together with the development of mass spectrometers with a fast duty cycle, increased sensitivity and resolving power. Recent introduction of the orbitrap mass spectrometer, in particular the latest version (orbitrap Fusion Lumos, and its expected successor the Orbitrap Fusion Lumos $1 \mathrm{M}$ ) as well as search algorithms greatly facilitate this process.

The current methodology monitors the kinetic assembly and disassembly of anthrax toxin components using label-free BLI methodologies and evaluates the structure and identity of these components using EM and MS, respectively. The use of a simple single channel BLI system coupled with routine negative staining EM analysis and elementary MS techniques are more than adequate to characterize an assembly process. 


\section{Disclosures}

No disclosures at this time.

\section{Acknowledgements}

This work was supported by the Madison and Lila Self Graduate Fellowship (AJM), NIH 5T32GM008359 (PTO), KUMC Biomedical Research Training Program (PTO), NIH R01AI090085 (MTF), KU Bridging Funds and the Biomolecule Interaction Technologies Center (BITC) Grant (CT and MTF). The authors would like to thank Barbara Fegley at KUMC Electron Microscopy Core for assistance with TEM image acquisition.

\section{References}

1. Bellon, S., Buchmann, W., Gonnet, F., Jarroux, N., Anger-Leroy, M., Guillonneau, F., and Daniel, R. Hyphenation of surface plasmon resonance imaging to matrix-assisted laser desorption ionization mass spectrometry by on-chip mass spectrometry and tandem mass spectrometry analysis. Analytical Chemistry. 81, 7695-7702 (2009).

2. Kim, Y. E., Yi, S. Y., Lee, C. S., Jung, Y., and Chung, B. H. Gold patterned biochips for on-chip immuno-MALDI-TOF MS: SPR imaging coupled multi-protein MS analysis. The Analyst. 137, 386-392 (2012).

3. Abdiche, Y., Malashock, D., Pinkerton, A., and Pons, J. Determining kinetics and affinities of protein interactions using a parallel real-time label-free biosensor, the Octet. Analytical Biochemistry. 377, 209-217 (2008).

4. Abdiche, Y. N., Malashock, D. S., Pinkerton, A., and Pons, J. Exploring blocking assays using Octet, ProteOn, and Biacore biosensors. Analytical Biochemistry. 386, 172-180 (2009).

5. Pall. BLI Technology. <http://www.fortebio.com/bli-technology.html> (2018).

6. Naik, S., Brock, S., Akkaladevi, N., Tally, J., McGinn-Straub, W., Zhang, N., Gao, P., Gogol, E. P., Pentelute, B. L., Collier, R. J., and Fisher, M. T. Monitoring the kinetics of the $\mathrm{pH}$-driven transition of the anthrax toxin prepore to the pore by biolayer interferometry and surface plasmon resonance. Biochemistry. 52, 6335-6347 (2013).

7. Akkaladevi, N., Hinton-Chollet, L., Katayama, H., Mitchell, J., Szerszen, L., Mukherjee, S., Gogol, E., Pentelute, B., Collier, R., and Fisher, M. Assembly of anthrax toxin pore: Lethal-factor complexes into lipid nanodiscs. Protein Science. 22, 492-501 (2013).

8. Jin, H., Cantin, G. T., Maki, S., Chew, L. C., Resnick, S. M., Ngai, J., and Retallack, D. M. Soluble periplasmic production of human granulocyte colony-stimulating factor (G-CSF) in Pseudomonas fluorescens. Protein Expression and Purification. 78, 69-77 (2011).

9. Yamniuk, A. P., Edavettal, S. C., Bergqvist, S., Yadav, S. P., Doyle, M. L., Calabrese, K., Parsons, J. F., and Eisenstein, E. ABRF-MIRG benchmark study: Molecular interactions in a three-component system. Journal of Biomolecular Techniques : JBT23. 101-114 (2012).

10. Moore, J. D., Perez-Pardo, M. A., Popplewell, J. F., Spencer, S. J., Ray, S., Swann, M. J., Shard, A. G., Jones, W., Hills, A., and Bracewell, D. G. Chemical and biological characterisation of a sensor surface for bioprocess monitoring. Biosensors \& Bioelectronics. 26, 2940-2947 (2011).

11. Feld, G. K., Thoren, K. L., Kintzer, A. F., Sterling, H. J., Tang, II, Greenberg, S. G., Williams, E. R., and Krantz, B. A. Structural basis for the unfolding of anthrax lethal factor by protective antigen oligomers. Nature Structural \& Molecular Biology. 17, 1383-1390 (2010).

12. Fairhead, M., and Howarth, M. Site-specific biotinylation of purified proteins using BirA. Site-Specific Protein Labeling: Methods and Protocols. 171-184 (2015).

13. Naik, S., Kumru, O. S., Cullom, M., Telikepalli, S. N., Lindboe, E., Roop, T. L., Joshi, S. B., Amin, D., Gao, P., and Middaugh, C. R. Probing structurally altered and aggregated states of therapeutically relevant proteins using GroEL coupled to bio-layer interferometry. Protein Science. 23, 1461-1478 (2014).

14. Bandyopadhyay, A., and Gao, J. Iminoboronate-based peptide cyclization that responds to $\mathrm{pH}$, oxidation, and small molecule modulators. Journal of the American Chemical Society. 138, 2098-2101 (2016).

15. Grantham, J., Llorca, O., Valpuesta, J. M., and Willison, K. R. Partial occlusion of both cavities of the eukaryotic chaperonin with antibody has no effect upon the rates of beta-actin or alpha-tubulin folding. The Journal of Biological Chemistry. 275, 4587-4591 (2000).

16. Ercius, P., Alaidi, O., Rames, M. J., and Ren, G. Electron Tomography: A Three-Dimensional Analytic Tool for Hard and Soft Materials Research. Advanced Materials. 27, 5638-5663 (2015). 